Itinéraires Itinéraires

Littérature, textes, cultures

\title{
Le DVD comme vecteur d'une culture du chapitre en contexte médiatique
}

Quelques manifestations d'une énonciation chapitrale

DVD Publishing as a Vehicle of a Culture of the Chapter in a Media Context.

Chaptering as Enunciation

Alain Boillat

\section{OpenEdition}

Journals

Édition électronique

URL : http://journals.openedition.org/itineraires/7956

DOI : $10.4000 /$ itineraires. 7956

ISSN : 2427-920X

Éditeur

Pléiade

Référence électronique

Alain Boillat, "Le DVD comme vecteur d'une culture du chapitre en contexte médiatique », Itinéraires [En ligne], 2020-1 | 2020, mis en ligne le 02 octobre 2020, consulté le 14 novembre 2020. URL : http:// journals.openedition.org/itineraires/7956 ; DOI : https://doi.org/10.4000/itineraires.7956

Ce document a été généré automatiquement le 14 novembre 2020.

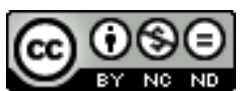

Itinéraires est mis à disposition selon les termes de la licence Creative Commons Attribution - Pas d'Utilisation Commerciale - Pas de Modification 4.0 International. 


\title{
Le DVD comme vecteur d'une culture du chapitre en contexte médiatique
}

\author{
Quelques manifestations d'une énonciation chapitrale
}

DVD Publishing as a Vehicle of a Culture of the Chapter in a Media Context. Chaptering as Enunciation

Alain Boillat

1 Principalement associé à la pratique romanesque, le chapitrage a connu avec la diffusion de films sur disques optiques (LaserDisc, DVD puis Blu-ray) un essor considérable au sein de la culture médiatique des deux dernières décennies, tout en constituant paradoxalement une forme d'impensé dans le domaine des études cinématographiques, où le support a été massivement utilisé à des fins de consultation et d'enseignement tout en ne faisant guère l'objet, en lui-même, d'investigations théoriques. Au tournant du xxi siècle, avant que l'usage du DVD ne se banalise, de nombreux commentaires furent toutefois émis à propos de cette nouvelle opportunité de visionnage, parfois relatifs au principe de l'indexation du contenu filmique en chapitres. Faisant le point sur la chronique de Nicolas Saada dans Les Cahiers du cinéma initiée en janvier 2000 (et intitulée «Le lecteur de DVD» à partir d'avril), André Chaperon notait, à une époque où l'acquisition de galettes DVD visait davantage un retour sur une projection en salles que la découverte d'un film, que «le lecteur DVD (l'appareil) est ainsi utilisé comme une instance de vérification, comme l'auxiliaire d'une cinéphilie fétichiste fonctionnant selon une politique du morceau choisi, exhibé comme une preuve sur le corps morcelé d'un film qui n'a même plus à être dans une relation organique avec ce qu'on a extrait de lui " (Chaperon 2001b : 33); se référant ensuite aux écrits de Serge Daney sur le régime de la consommation télévisuelle, Chaperon soulignait combien certains « cinéastes post-publicitaires» tels que Beineix ou Besson avaient en quelque sorte anticipé dans leur pratique filmique même ce type de lecture fragmentée dont René Barjavel, dans un essai d'anticipation paru en 1944, imaginait de manière spéculative l'existence ${ }^{1}$. Par ailleurs, les contributions de 
l'ouvrage collectif Film and Television after DVD (Bennett et Brown 2008a) mettent en évidence le changement de paradigme induit par la location et la vente de films sur support DVD qui a connu une pénétration phénoménale du marché domestique; il s'agissait, avec quelque 84 millions de foyers équipés d'un lecteur DVD en 2005 aux États-Unis, de "l'article le plus vendu de l'histoire du marché américain de l'électronique grand public ${ }^{2} »$. À notre époque où ce support physique propice à l'exercice du fétichisme cinéphilique est menacé d'obsolescence en raison de la concurrence que représentent notamment la VOD, le téléchargement et le streaming, il nous semble productif d'envisager ce phénomène historique et culturel à l'aune des recherches conduites dans le domaine des études littéraires telles qu'elles se sont développées à la suite des travaux d'Ugo Dionne, en partant du constat suivant, formulé en conclusion de l'ouvrage collectif Pratiques et poétiques du chapitre: «la poétique du chapitre est [...] articulée à une "poétique du support", qui imbrique l'étude des formes narratives dans une réflexion sur leur inscription énonciative et médiatique » (Colin, Conrad et Leblond $2017: 332$ ).

2 Il s'agit dans le présent article, à partir d'exemples variés et dans une optique plus exploratoire que systématique - ainsi nous ne circonscrirons pas de corpus défini en fonction, par exemple, d'une pratique éditoriale spécifique -, d'esquisser quelques axes généraux pour appréhender le chapitrage des DVD, en passant, à partir des observations d'Ugo Dionne, non seulement d'un moyen d'expression à un autre (de la littérature au cinéma), mais aussi en changeant de support physique. En effet, livre et DVD sont le fruit d'un travail d'édition postulant le choix d'un dispositif chapitral donné. Notre attention se portera par conséquent moins sur le film même - ce qui impliquerait de discuter en outre les formes de chapitrage internes au discours filmique et visibles également à l'écran lors d'une projection en salles ${ }^{3}$ - que sur le support de diffusion du média (même si une coïncidence peut, comme nous le verrons, être établie dans certains cas entre l'un et l'autre), et moins sur les conditions pratiques d'un travail éditorial (qui mériterait néanmoins une étude en soi dans une perspective sociohistorique $)^{4}$ que sur les enjeux théoriques - en particulier discutés en termes d' énonciation filmique - des modalités selon lesquelles un DVD (chapitré) programme la lecture du film dont il contient une copie. La projection d'un film impose au spectateur une linéarité de la lecture (sur laquelle agit en dernière instance le projectionniste), de sorte que la notion de "temps du récit» (par opposition au "temps de l'histoire») proposée par Gérard Genette (1972) pour étudier les œuvres romanesques s'applique d'autant mieux à tout film (narratif ou non), dont le métrage (pour une même cadence de projection) équivaut à une durée effective - le temps "écranique " que les filmologues déjà distinguaient du temps diégétique (voir Gaudreault et Jost 1990 : 116-117) -, alors que le critère du nombre de pages d'un roman correspond à une temporalité susceptible de présenter des fluctuations importantes en fonction des lecteurs. Le visionnage domestique d'un film permet quant à lui à l'usager de se réapproprier une forme de contrôle sur la temporalité de son expérience, non seulement en pressant sur " pause ", mais aussi en revenant au menu pour sélectionner à n'importe quel moment une scène précédente ou suivante, c'est-à-dire en opérant un choix parmi des possibilités de balisage du film qui, dans le cas du DVD (contrairement au parcours très libre sur une timeline proposé par la lecture d'un film sur le disque dur du décodeur d'une chaîne câblée par exemple), sont prédéterminées par l'éditeur et exhibées grâce à différents modes de visualisation. 


\section{L'instance d'énonciation chapitrale}

3 Composante d'une pratique éditoriale plus large, le chapitrage, qui, au cinéma plus encore qu'en littérature, n'est presque jamais le fait des auteurs de l'œuvre diffusée, consiste en la présentation d'une segmentation d'un texte - en l'occurrence ici d'un texte filmique - dont les différents morceaux, situés à un niveau supérieur à celui du plan et s'apparentant à une "scène " ou à une "séquence " sans pour autant se confondre avec ces unités définies en termes d'espace, de temps et/ou d'action (le chapitre subsume souvent plusieurs séquences), exhibent une pratique de la segmentation qui affleure parfois dans le film sous la forme de signes de ponctuation filmiques - pour reprendre l'expression de Christian Metz (1972), qui inclut dans cette catégorie les volets, fondus, noirs, etc. - et dont on observe l'omniprésence à tous les stades de la fabrication du film, de l'écriture (en particulier au stade du «découpage technique $\left.{ }^{5} »\right)$ au montage, en passant par le tournage (défilement photogrammatique, succession des prises de vue). Il est à noter que Metz lie la question de la ponctuation à celle de la démarcation, ce qui le conduit à comparer certaines formes d'interruption de l'image à l'écran avec la rupture produite par le chapitrage d'un roman, pointant ainsi la composante matérielle et visible de ce dernier :

Si les parenthèses et les guillemets que l'on trouve dans les livres sont des signes partiellement arbitraires, le point qui sépare deux phrases [...], le passage à la page qui sépare deux chapitres donnent lieu plus nettement, comme dans les films de fiction romanesques, à des phénomènes d'induction diégétique qui favorisent la "ressemblance" entre le signifié [...] et le signifiant [...]. Cette page, qui reste blanche à la fin du chapitre, sépare moins brutalement que ne le font les vingt minutes d'entracte (de plus, ce n'est pas forcément à cette page-là que les lecteurs choisiront d'interrompre la lecture, si du moins ils l'interrompent). (Metz 1972 : 115-116)

Le DVD, lui, offre une situation médiane entre l'imposition de moments de rupture par l'instance éditoriale et le choix de ces moments par le spectateur. Ces deux pôles ne coïncident toutefois pas nécessairement, comme le souligne le commentateur du DVD (Montparnasse, 2001) du film Johnny Guitar (1954) à propos d'une scène devenue culte : « Entre l'invitation de Vienna à Johnny [...] et son revirement soudain [...], on avait un chapitre idéal [...] que l'on aurait regardé indéfiniment. Mais non, le chapitrage commence beaucoup trop en amont, et s'arrête avec la dispute avec Bart [...], comme si cette dispute méritait d'être chapitrée » (Delorme 2001: 45). On voit bien que les critères de la démarcation touchent à des aspects narratifs, mais aussi plus généralement à la construction de la culture cinéphilique qui incite à voir dans ce passage de ce western de Nicolas Ray une forme de "modernité ». Les possibilités récentes offertes par le visionnage de films stockés sous forme de fichiers numériques pourraient en partie changer la donne. Ainsi, pour rester dans le domaine de l'édition de disques, l'option «Mes scènes " proposée sur certains Blu-Ray Universal selon une logique parente de celle des signets de la navigation sur le Web permet à l'usager d'enregistrer sur une frise temporelle des points d'entrée et de sortie délimitant des scènes qu'il a lui-même choisies; ce type d'interactivité induirait dès lors la suppression de l'instance chapitrale si un découpage « officiel » n'était pas par ailleurs proposé (contrairement à des DVD gravés dont l'usager indexe lui-même le contenu).

4 Le chapitrage est plus largement soumis à une tension entre continuité et discontinuité qui se trouve au principe de l'appareil cinématographique. En effet, lorsque les éditeurs de DVD qui agrémentent grâce à des illustrations l'écran de présentation - ce « menu » 
qui permet de privilégier le tabulaire sur le linéaire au même titre que la page d'un nouveau chapitre peut comprendre dans un roman divers ornements - et optent pour une identification individuelle de chaque chapitre, ils sont significativement confrontés à un choix portant soit sur une image fixe emblématique d'un chapitre donné (parfois assortie d'une musique et/ou d'un dialogue entendu de manière répétée qui renforce l'inscription du segment filmique dans la durée), soit sur un court extrait en mouvement (sonore ou non) qui défile en boucle. Ce renvoi métonymique au contenu de l'ensemble d'un chapitre par le biais d'une citation (audio)visuelle - parfois élue pour son statut emblématique au sein du segment ${ }^{6}$, parfois correspondant simplement à l'entame de l'extrait ainsi délimité - exhibe la nature fragmentée du discours filmique, et instaure un rapport entre titrage et contenu du chapitre dont on ne peut trouver véritablement d'équivalent dans un roman, à moins que le titre ne reprenne exactement une phrase qui apparaît plus tard dans le texte. Un tel cas de figure, situé au seul niveau du langage verbal, se rencontre d'ailleurs dans les titres de chapitres proposés par les éditeurs de DVD, qui intitulent fréquemment un ou plusieurs des chapitres délimités en reprenant entre guillemets une courte réplique saillante du passage en question ${ }^{7}$. Cette pratique, corrélative de l'importance conférée au travail du dialoguiste dans le cinéma populaire du patrimoine français ${ }^{8}$, a pour conséquence d'atténuer la présence dans le sommaire du menu d'une instance narrative qui ressaisirait en une formule synthétique l'ensemble du contenu actionnel et dialogique d'une partie délimitée du film. Il n'en demeure pas moins que tout film chapitré sur support DVD peut être envisagé à travers un niveau énonciatif supplémentaire à ceux identifiés aux matières de l'expression filmique (voir Boillat 2007 : 381-447). On pourrait qualifier ce niveau d'« énonciation chapitrale", perceptible de manière facultative lorsque l'usager s'enquiert des informations livrées par l'éditeur. La granularité du découpage, qui peut s'étendre de moins de dix chapitres à une cinquantaine (dans le cas de «films cultes» dont chaque séquence ou presque est devenue célèbre, à l'exemple des productions de la franchise Star Wars $^{9}$ ), a une incidence sur la lecture du film chapitré. Ainsi, par exemple, une plus grande fragmentation s'avère moins contraignante pour un usager qui est à la recherche d'un passage donné, tandis que le degré de clarté (ou respectivement d'opacité) des titres influe potentiellement sur le savoir du spectateur, même si en général le chapitrage n'est consulté qu'à partir du deuxième visionnage du film.

5 La présence de cette instance est d'autant plus marquée que le chapitrage s'accompagne très majoritairement, à un niveau écranique (menu du DVD) et/ou périfilmique $^{10}$ (jaquette, livret, emballage cartonné, etc.) de mentions écrites, fussentelles réduites à des nombres. Ce retour de l'écrit rappelle les cartons du cinéma muet ${ }^{11}$ (utilisés encore aujourd'hui, justement, pour indiquer dans le discours filmique même le titre d'un chapitre) ainsi que le sous-titrage, favorisé par le DVD en raison de la mise à disposition du film en plusieurs langues (sous-titrage et doublage, voire ajout d'un commentaire over sur l'ensemble du film ${ }^{12}$ ). Comme dans un roman, la mention des chapitres engage une dimension typographique et topographique (un lieu donné dans le déroulement du film et sur la surface de l'écran). Le spectateur est dans ce cas placé en position de lecteur, ce qui n'est pas sans incidences, de façon plus générale, sur sa lecture du film, les chapitres pouvant être considérés, comme l'a noté Dionne, comme «des extraits visuels, des morceaux soumis à l'analyse » auxquels est accordée de facto par l'usager une certaine " cohérence sémantique » (Dionne $2008: 12$ ). Dans le cas d'un chapitrage de DVD effectué consciencieusement (ce qui est l'apanage d'une minorité d'éditeurs), le choix de la segmentation et les titres choisis résultent d'une opération 
qui peut être considérée comme une première étape analytique. Derrière la liste des intitulés peut en effet apparaître en filigrane la structure narrative du film, et cela d'autant plus si l'instance chapitrale s'appuie sur des règles de construction du récit, ou du moins met en exergue celles qui ont présidé à l'écriture du scénario du film.

\section{1 : l'odyssée du DVD - premières pistes}

6 La construction théorique d'une instance d'énonciation chapitrale postule la mise en place par l'éditeur de stratégies spécifiques présidant à l'opération de chapitrage. Une étude d'un corpus de type «Big Data " reste à faire pour repérer des tendances, les historiciser et en établir une typologie. Une telle recherche pourrait s'appuyer sur la base de données en ligne "ChapterDb.org", destinée à fournir aux utilisateurs de fichiers mp4 les intitulés des chapitres issus de disques (DVD, Blu-ray, HD-DVD), ces derniers n'apparaissant pas dans les titres de fichiers. L'existence même de cette base de données dit bien l'intérêt suscité par le chapitrage dans les médias audiovisuels actuels.

7 Cependant, les revues spécialisées dans la critique de DVD, dont certaines étaient initialement consacrées à la VHS puis se sont adaptées aux supports numériques ${ }^{13}$, ne font guère mention du chapitrage, auquel n'est associé aucun paramètre dans le système d'évaluation des chroniqueurs. L'accent est prioritairement mis dans ces articles sur la qualité de l'image et du son - selon une démarche technophile ${ }^{14}-$, ainsi que sur l'intérêt que représentent les suppléments. En général, les fiches techniques comprennent des abréviations qui renvoient à différents aspects du film commenté (format, sous-titre, standards sonores, etc.), mais aucune d'elles ne concerne le chapitrage. D'après ce qu'un relevé non systématique nous donne à penser, la mention de chapitres est utilisée à des fins strictement pratiques lorsque le journaliste se réfère à un passage donné du film, afin que le lecteur puisse se rendre rapidement au passage discuté $^{15}$. Une étude plus approfondie de ce type de sources qui témoignent de la réception des films en DVD (Ultimate DVD, DVD Monthly, DVDvision, Les Années Laser, etc.) permettrait de dégager d'éventuels discours sur le chapitrage, même si on peut préjuger de la faible attention accordée à cette question. Ainsi, sur le site des Années Laser, une recherche à l'aide du mot-clé " chapitrage », certes menée uniquement sur des textes en ligne récents, ne débouche que sur deux entrées, toutes deux relatives à une critique de la faible interactivité du Blu-ray en raison de l'absence d'accès à un chapitrage ${ }^{16}$. Ce dernier va à ce point de soi que seule son absence lui vaut une mention.

8 Il en va par contre autrement de la période comprise entre 2000 et 2006, que l'on pourrait qualifier de véritable "moment DVD", non seulement en termes de rentabilité de ce marché (avec, pour le marché français qui nous intéresse ici, un décollage des ventes en 2001, un sommet en 2004 et une première chute significative en $2006^{17}$ ), mais aussi d'affirmation d'un discours prônant une certaine ambition éditoriale. La publication consécutive en 2001 et 2002 par les Cahiers du cinéma de deux numéros hors-série présentés comme des guides pour l'achat de DVD peu avant les fêtes de fin d'année est révélatrice de l'essor momentané des discours relatifs à ce nouveau support de diffusion de films ${ }^{18}$. Dans l'éditorial d'un numéro hors-série de DVDvision paru en mai 2001 et consacré à l'édition de Lawrence d'Arabie (1962), le comédien britannique Peter O'Toole émet le constat suivant: "Nous sommes maintenant au DVD qui marque la fin du visionnage linéaire du film et le triomphe du 
chapitrage, avec le choix direct de votre séquence préférée » (O’Toole $2001: 3$ ). Il s'agit de prendre la mesure de l'enthousiasme suscité au tout début du xxI siècle par le disque optique dans le domaine du cinéma. Contrairement à la cassette VHS, qui postulait quant à elle, à l'instar de la pellicule de cinéma (y compris dans ses usages " amateurs » à domicile), un visionnage défini par un principe du défilement continu (l'avance rapide et le rembobinage n'y étaient guidés que par le timecode), les formats vidéo numériques qui se sont généralisés grâce au DVD se caractérisent principalement par une interactivité accrue en raison d'un dispositif conçu dès les années 1950 pour la télévision : la télécommande. L'usager a dès lors notamment la possibilité d'accéder aisément à des points prédéterminés du film, à l'instar du lecteur d'un roman chapitré qui peut identifier les parties du texte en feuilletant l'ouvrage ou en consultant un sommaire. Ce changement de paradigme, que l'on peut comparer, toutes proportions gardées, au rôle qu'a joué, dans l'histoire de l'écriture, le passage du rouleau au codex, valorise les unités pour elles-mêmes, et par conséquent favorise la mise en place d'un dispositif chapitral.

Le marché qui s'est ainsi ouvert avec le DVD a permis l'émergence d'un travail éditorial comportant de nombreuses composantes qui vont de la restauration du film numérisé (voire de la mise à disposition de versions dites "director's cut » spécifiquement prévues pour ce support) à l'ajout de divers «bonus ${ }^{19}$ » en passant, justement, par l'établissement d'un chapitrage (relatif au film, mais parfois aussi aux suppléments ${ }^{20}$ ). Cette pratique s'explique par la nature du support - le CD musical, apparu avant le DVD, est lui aussi généralement divisé en plages auxquelles sont associés numéros et titres sur la jaquette de la boite, et d'ailleurs une version du DVD de Django Unchained offre un double accès aux scènes du film, soit par un sommaire comprenant 28 segments numérotés et titrés, soit par une liste de l'ensemble des 38 morceaux de musique qui parsèment la bande sonore ${ }^{21}-$, mais aussi par un changement de stratégie éditoriale qui, originellement basée sur le système de la location de VHS, a rapidement privilégié la vente à très grande échelle, l'achat se justifiant entre autres, parallèlement au développement du marché des installations de home cinema, par le confort de visionnages répétés et partiels dont le chapitrage contribue à assurer le balisage. La valorisation du fragment, des systèmes audio et de scènes spectaculaires regorgeant d'effets visuels qui résulte de l'exacerbation de la dimension attractionnelle (plutôt que narrative ${ }^{22}$ ), en particulier dans le cas du cinéma hollywoodien qui mise depuis la seconde moitié des années 1970 sur la production de blockbusters faisant office de vitrine technologique (l'exemple le plus emblématique est Avatar de James Cameron, 2009 , dont la diffusion a notamment servi de promotion à la vente de Blu-ray et de téléviseurs 3D) ${ }^{23}$. Dans une interview parue en décembre 2001, on demande à Christophe Gans s'il ne craint pas «qu'avec le système des chapitres on évite de se laisser porter par les moments de latence "; le cinéaste concède qu'à cette époque, «des gens achètent des DVD pour leurs performances techniques, pour montrer leur supermatos à leurs copains", tout en affirmant que "les vrais amateurs de cinéma regardent les films » (Cohen et Chauvin 2001: 86) - la formule "regarder un film» supposant ici un visionnage continu impliquant notamment un travail de configuration narrative. De telles prises de parole révèlent combien la question du chapitrage s'inscrit plus largement d'une part dans des dispositifs technologiques dont la variété s'est aujourd'hui accrue avec la diversification des écrans, d'autre part dans des pratiques sociales de consommation de productions médiatiques. 
10 L'année 2001 correspond à une période où le DVD est apprivoisé par la critique cinéphilique, pour laquelle le chapitrage ne relève pas encore de l'évidence. Si le découpage en chapitres ne constitue sans doute pas un argument de vente décisif pour ce type de support ni n'est, dans les faits, nécessairement exploité par l'usager, il n'en demeure pas moins qu'avant l'essor du Blu-ray entre 2006 et $2010^{24}$, la présence d'un chapitrage faisait souvent l'objet d'une mention sur la jaquette du DVD, tandis que les modalités de sa présentation contribuaient à donner un indice du degré d'investissement de l'éditeur dans la confection du produit (à partir du degré zéro que représente la plage unique ou une segmentation automatique à intervalle régulier ${ }^{25}$ ). Par conséquent, le chapitrage a pu être considéré comme une forme de plus-value. Il est intéressant de lire dans le premier numéro hors-série des Cahiers du cinéma de décembre 2001 le commentaire d'une journaliste qui, à partir du visionnage du DVD du film El (Luis Buñuel, 1952), fait à propos de sa première expérience avec ce format l'observation suivante :

Au regard de l'autre, Buñuel élève des murs, des remparts, des forteresses, des colonnes, il sépare ce qui doit être protégé de ce qui le menace [...]. Ce qui m'a peutêtre conduit [sic] à regarder $E l$ de cette façon, c'est aussi le chapitrage. C'est une notion que je découvre. Elle m'a paru très littéraire, comme si les scènes d'un film pouvaient être présentées tels les chapitres d'un livre. Mais ici, ils ne sont pas disposés de façon linéaire, dans une suite verticale chronologique et numérotée mais un peu comme des tableaux accrochés sur un mur, que l'on pourrait décrocher comme des objets en soi. [...] présenté de cette façon, le film est un objet qu'on peut facilement découper, dont on peut isoler une partie sans savoir où elle se situe dans le film. (Loiseleux $2001: 26$ )

Cette utilisatrice d'un lecteur DVD qui visionne pour la première fois un film dans ce format établit une corrélation entre deux niveaux de lecture du film, d'une part l'interprétation de l'œuvre mexicaine de Buñuel, d'autre part les modalités de son visionnage par le truchement d'une interface. On constate qu'en 2001, le chapitrage ne va pas de soi, puisqu'il est décrit comme l'objet d'une découverte, d'une expérience nouvelle dont l'auteure rend compte en recourant à des références à d'autres pratiques artistiques (littéraire et picturale). Si elle utilise la comparaison avec des «tableaux accrochés sur un mur ", c'est que chaque page du menu de $E l$, accompagné de musique, comporte cinq vignettes correspondant chacune à un chapitre (dix au total). La linéarité et la chronologie qui sont ici relevées en raison d'une exploitation de la dimension tabulaire de l'écran sont à nuancer, puisque, dans l'édition concernée (Films sans frontières, "collection Auteurs", 2001), chaque chapitre est pourvu d'un matricule - élément que l'on sait «voué tout entier à [la] fonction de repérage et de désignation» (Dionne 2008: 366) - et d'un titre, ne laissant aucun doute sur la chronologie des fragments. En outre, la comparaison avec le livre ne s'effectue pas tant sur un plan matériel (tout livre, même un recueil de recettes de cuisine, peut faire l'objet d'un chapitrage) que symbolique (la pratique serait «très littéraire»), le chapitrage participant alors à une démarche de légitimation culturelle du produit filmique. Les auteurs de l'éditorial des Cahiers du cinéma soulignent ce même point :

Du livre, il tient le découpage en chapitres, la présence d'éditeurs ou encore les bonus qui, parfois, semblent une réminiscence des fameuses notes de bas de page. Et l'on imagine qu'à terme, le DVD aura ses livres de poche, ses "pléiades", ses éditions de luxe et limitées. (Jousse et Larcher $2001: 6$ )

Bien que situant le DVD entre livre et disque, Thierry Jousse et Jérôme Larcher n'identifient en rien le chapitre à un morceau de musique (en dépit de sa parenté évidente avec une plage de CD musical). Le modèle est résolument littéraire, et se 
décline en formats éditoriaux définis sans référence aucune aux standards de la distribution cinématographique, le film étant devenu, de fait, un objet de «librairie ». Dans le texte introductif reproduit dans les livrets des DVD de la série « Revoir François Truffaut " placée sous la direction de Serge Toubiana avec laquelle MK2 s'insère en 2001 sur ce marché, Marin Karmitz recourt à la même référence, et répond en quelque sorte à la supputation des éditorialistes des Cahiers du cinéma (dont Toubiana fut autrefois le directeur):

Aujourd'hui, mk2 voit dans les possibilités offertes par l'innovation technologique du DVD l'opportunité de prolonger son activité. En lançant la collection mk2, nous avons l'ambition d'aborder l'édition DVD en s'inspirant de «La Pléiade » avec ses préfaces et ses notes. (Livret MK2)

11 Le chapitrage procède ainsi d'une intention plus large de proposer une plus-value grâce à un travail éditorial consciencieux ${ }^{26}$ - certaines boîtes enfermaient même un questionnaire à retourner par les acheteurs afin qu'ils puissent manifester leur intérêt pour tel ou tel aspect du produit (en l'occurrence, le chapitrage n'y était pas mentionné) -, et se voit par conséquent mis en évidence dans le péritexte, où il en est fait mention à plusieurs reprises. Au verso de la boîte cartonnée du DVD du Vent nous emportera (Abbas Kiarostami, 1999), le contenu du premier des deux DVD (MK2, 2002) est présenté sous la forme de deux composantes mentionnées dans un même format et affublées d'une pastille identique: «•Le film; •Chapitrage». Évidemment, le film apparaît en premier en tant qu'objet principal auquel se rapporte le second point, mais le choix typographique suggère presque une égalité entre ces deux "prestations " livrées par le produit. Par ailleurs, sur ce DVD comme sur d'autres édités par MK2 à la même période ${ }^{27}$, le chapitrage est mis en exergue à un niveau écranique où il est affiché, selon le choix de l'usager, selon deux modalités : il s'agit soit d'une liste qui se déploie sur plusieurs écrans successifs pendant que se fait entendre la bande-son des passages correspondants, soit d'une image plein cadre illustrant chacun des chapitres. L'interactivité définit donc le mode d'accès même à cette fonctionnalité du menu. Dans le cas du DVD de Madame Bovary de Claude Chabrol (1991), film dans lequel l'hypotexte romanesque est d'autant plus important que certains passages du livre sont cités littéralement en voix over, chaque chapitre, lui-même inscrit au sein d'une partie, est désigné par un matricule assorti d'une phrase verbale conjuguée au passé simple (ou d'une phrase interrogative/exclamative en style direct) citée de la prose de Flaubert qui permet d'identifier les articulations clés du récit. Le principe d'établissement de ces titres thématiques s'effectue par analogie avec la pratique qui consiste à répertorier une œuvre littéraire sans titre par son incipit. Une phrase telle que « Un matin, le père Rouault vint apporter à Charles le montant de sa jambe remise " ouvre précisément l'un des chapitres du roman, en l'occurrence ici le chapitre 3 (qui correspond au deuxième de l'édition du film dans la mesure où le premier chapitre consacré à la jeunesse de Charles a été supprimé dans l'adaptation de Chabrol). Contrastant avec l'absence de titres de chapitre qui caractérise le roman de Flaubert, le paratexte proposé dans l'édition DVD de Mme Bovary en vient ainsi à sursignifier la dimension romanesque de l'adaptation cinématographique. Plus encore que dans la plupart des DVD chapitrés, où la «maniabilité généralisée augmente, lors du visionnement, la présence du dispositif, tout en diminuant [...] le potentiel de visibilité [des] objets filmiques » (Le Forestier $2010: 163)^{28}$, les possibilités de navigation au sein d'un contenu audiovisuel s'affichent avec un chapitrage de ce type. 


\section{L'apax lynchien : le chapitre comme clé de lecture} propos d'occurrences qu'il s'agirait dans une étude plus vaste d'envisager dans leur diachronie) correspond également à la sortie du film Mulholland Drive de David Lynch, dont l'édition DVD par StudioCanal (2002), plus largement inscrite dans une exploitation par la chaîne Canal+ de l'engouement exégétique hors du commun provoqué auprès du public par la sortie du film ${ }^{29}$, présente la particularité fort rare ${ }^{30}$ (puisque les lecteurs DVD, pensés pour donner accès à un récit, ne disposent pas de la touche «random» de certains lecteurs CD contemporains) de proposer un chapitrage supposément établi en concertation avec le cinéaste et reposant sur un accès aléatoire aux scènes sélectionnées (à chaque entrée de chapitre sont associés entre trois et six extraits ${ }^{31}$, l'actualisation de l'un d'eux étant indépendante de l'utilisateur). Dans une image assez dense représentant l'intérieur d'un appartement, l'usager doit, comme dans un jeu vidéo d'aventure, sélectionner certains objets issus du film sur lesquels il peut cliquer pour voir démarrer un chapitre, ou du moins un extrait de film qui entretient un lien avec l'objet représenté. L'objectif de l'éditeur est de maintenir l'opacité du récit filmique et de favoriser l'activité de décodage, le péritexte éditorial du DVD prenant soin de préciser que le chapitrage ne déflore pas, dans ce cas, le plaisir interprétatif suscité par le film.

La pratique sera reconduite et même complexifiée pour le long-métrage suivant du cinéaste, Inland Empire (2006), dont la jaquette du DVD, qui valorise à l'extrême le film dans une perspective auteuriste ${ }^{32}$ (le texte placé en titre du verso est «La Joconde de David Lynch »), annonce un " chapitrage aléatoire par mots-clés, créé par Michel Chion d'après son livre sur David Lynch» (StudioCanal, 2007). Même si le terme de « chapitre » n'apparaît plus à l'écran lorsqu'on accède à cette fonctionnalité qualifiée de "Lynch-kit », aucun chapitrage ne revendique à notre connaissance comme celui-ci un tel ancrage dans une analyse filmique préalable, perceptible à l'écran en ce que l'usager peut cliquer sur un astérisque pour accéder à une définition de chacun des termes-clés en lien avec le «monde» de Lynch ${ }^{33}$.

\section{Un palimpseste de dispositifs chapitraux}

14 Nous avons discuté plusieurs caractéristiques de la pratique du chapitrage des éditeurs de DVD au moment de l'essor du support, au cours des toutes premières années du xxI ${ }^{\mathrm{e}}$ siècle, en constatant qu'elles n'ont guère évolué, le travail éditorial ayant plutôt tendance à régresser en dehors du marché de la restauration d'œuvres du patrimoine.

Un dernier point nous paraît d'importance dont nous proposons d'amorcer l'étude en conclusion : il s'agit d'une forme de superposition (ou de concurrence) potentielle entre l'instance d'énonciation du discours filmique et l'instance d'énonciation chapitrale de l'édition DVD. Elle survient lorsque le chapitrage du disque reprend (ou au contraire occulte) une segmentation plus ou moins explicitement présente dans le film. Une approche comparative peut en effet porter sur diverses éditions d'un même film, sur le chapitrage du DVD et celui de l'œuvre littéraire adaptée (comme nous l'avons fait cidessus avec Madame Bovary ${ }^{34}$ ) ou, justement, entre le périfilmique de l'édition DVD et le filmique. 

préalable. Il peut s'agir en effet de productions sérielles dans lesquelles la pratique relève du paradispositif selon Dionne (2008: 85-93), à l'instar de l'utilisation du terme «chapitre » pour qualifier les épisodes de serials tels que Flash Gordon dans l'aprèsguerre ou d'une série télévisuelle contemporaine prestigieuse comme House of Cards ${ }^{35}$; de tout film proposant un dispositif «chapitral » propre ${ }^{36}$ qui est repris par l'éditeur du DVD, soit de manière partielle - sur le disque des 8 salopards (Ascote Elite, 2015), le chapitrage du DVD ne se confond avec celui du film qu'à partir du chapitre 8 , qui débute avec le carton de titre du chapitre $4 \mathrm{du}$ film - ou systématique, comme cela est le cas dans Elephant (MK2, 2008) ${ }^{37}$, Valhalla Rising (Wild Side, 2009) ou Le Redoutable (StudioCanal, 2017) ${ }^{38}$, où le nombre d'unités est toutefois plus important dans le DVD que dans le film (la fonctionnalité du chapitrage diffère du visionnement en salles); enfin, les démarcations en chapitres peuvent participer d'un quasidispositif (Dionne 2008), en l'espèce, par exemple, de seuils introduits dans le film par l'insertion de segments narratifs enchâssés ou d'une structure fonctionnant par succession de sketchs ou de morceaux chantés et/ou chorégraphiés. Les bornes reprises du film pour le chapitrage du DVD peuvent être aussi moins ostensibles que l'usage « littéraire » de mentions écrites: dans le DVD de Dirty Harry (1971) édité par Warner notamment dont la première version cartonnée mettait très en évidence le chapitrage au verso du rabat de l'ouverture de la boîte -, plusieurs chapitres coïncident avec des points du film où interviennent des ellipses systématiques et singulières (en ce qu'elles conjuguent un caractère abrupte au niveau visuel tout en conservant une continuité au niveau du dialogue, la composante rythmique étant privilégiée sur la vraisemblance). Dans de tels cas, le chapitrage du DVD révèle et exhibe des pratiques de ponctuation interne au film (ou, au contraire, peut opter pour d'autres critères). Aussi, dans le DVD du Cheval de Turin (Béla Tarr, 2011) édité par Blaq Out en 2012, ce ne sont pas les cartons comportant la mention d'un jour précis qui sont retenus pour l'établissement de seuils, mais, dans un tel film constitué de longs plan-séquences "aboutés» (pour reprendre une terminologie utilisée par André Gaudreault à propos du cinéma des premiers temps), un principe qui consiste à comprendre en moyenne trois plans par chapitre, ce qui souligne l'organisation spatiale du film (en particulier l'opposition entre l'intérieur et l'extérieur de la ferme, avec des ponctuations où l'un des deux personnages vu de dos regarde par la fenêtre le paysage balayé par le vent).

De telles configurations dans lesquelles s'imbriquent deux niveaux de chapitrage (l'un redevable de l'instance de production du film, l'autre de l'éditeur du support numérique) demeurent encore à inventorier, à théoriser et, pour les plus intéressantes, à analyser au cas par cas en lien avec d'autres discours émis à propos des films et dans le contexte d'une véritable culture médiatique. Elles témoignent du rôle qu'endosse le « chapitrage » (au sens large) dans les productions télévisuelles et cinématographiques de ces vingt dernières années, et de l'importance accrue conférée à ce dernier à notre époque du numérique sur le plan non seulement des pratiques de montage, mais aussi de la diffusion. 


\section{BIBLIOGRAPHIE}

Barjavel, René, 1944, Cinéma total, Paris, Denoël.

Bennett, James et Brown, Tom, 2008a, Film and Television after DVD, New York, Londres, Routledge.

Bennett, James et Brown, Tom, 2008b, « Introduction: Past Boundaries of "New" and "Old" Media ", dans Film and Television after DVD, p. 1-18 (format Kindle).

Boillat, Alain, 2005, «L'éclatement du personnage chez Lynch », Décadrages, n 4-5, p. 48-63, [En ligne], DOI : 10.4000/decadrages.577.

Boillat, Alain, 2007, Du bonimenteur à la voix-over. Voix-attraction et voix-narration au cinéma, Lausanne, Antipodes.

Boillat, Alain, 2014, « Voice-over et voix over. Le dubbing comme objet des théories de l'énonciation filmique », dans A. Boillat et I. Weber Henking (dir.), Dubbing. Die Übersetzung im Kino / La traduction audiovisuelle, Marburg, Schüren.

Boillat, Alain, 2018, « Douze minutes avant minuit : l'effet "graphic novel" du chapitrage dans Watchmen ", Cahiers de Narratologie, $n^{\circ} 34$, [En ligne], DOI : 10.4000/narratologie.8757.

Brown, Tom, 2008, “"The DVD of Attractions?” The Lion King and the Digital Theme Park », dans J. Bennett et T. Brown, Film and Television after DVD, New York, Londres, Routledge, p. 80-100 (format Kindle).

Cahiers du cinéma, décembre 2001 (« Nos DVD, le guide des Cahiers »).

Chaperon, André, 2001a, « Plus fort que le DVD : le film », Hors-champ, nº 6, p. 27-31.

Chaperon, André, 2001b, « De la vérification extatique du fonctionnement des organes :

Télévision et DVD », Hors-champ, $\mathrm{n}^{\circ}$ 6, 2001, p. 33-35.

Charles, John, 1997, « Alice, Sweet Alice », Video Watchdog, nº 40, p. 46-47.

Cohen, Clélia et Chauvin, Jean-Sébastien, 2001, « Interview - Christophe Gans », Cahiers du cinéma, décembre 2001, p. 82-86.

Colin, Claire, Conrad, Thomas et Leblond, Aude, 2017, « Perspectives pour les chapitres suivants ", dans C. Colin, T. Conrad et A. Leblond, Pratiques et poétiques du chapitre du XIXe au XXI siècle, Rennes, PUR, p. 331-337.

Delorme, Stéphane, 2001, « Johnny Guitar de Nicholas Ray », Cahiers du cinéma, décembre 2001, p. 45.

Dionne, Ugo, 2008, La Voie au chapitre : poétique de la disposition romanesque, Paris, Seuil.

Gaudreault, André et Jost, François, 1990, Le récit cinématographique, Paris, Nathan.

Jousse, Thierry et Larcher, Jérôme, 2001, « Le film et l'objet », Cahiers du cinéma, décembre 2001, p. 6.

Genette, Gérard, 1972, Figures III, Paris, Seuil.

Genette, Gérard, 1987, Seuils, Paris, Seuil.

Gunning, Tom, 2006, «Le cinéma d'attraction : le film des premiers temps, son spectateur, et l'avant-garde », 1895. Mille huit cent quatre-vingt-quinze, $\mathrm{n}^{\circ}$ 50, [En ligne], DOI : 10.4000/1895.1242. 
Le Forestier, Laurent, 2010, «Le DVD, nouveau jouet optique? ", dans In the Very Beginning, at the Very End. Theories in Perspective, Udine, Forum, p. 159-166.

Le Forestier, Laurent, 2018, « Écrire le film : scénario/découpage en France (années 1920-1930) », dans A. Boillat et G. Philippe, L'Adaptation. Des livres aux scénarios. Approche interdisciplinaire des archives du cinéma français, Bruxelles, Les Impressions nouvelles, p. 41-68.

Loiseleux, Valérie, 2001, « Le DVD et moi », Cahiers du cinéma, décembre, p. 26.

McDonald, Paul, 2007, Video and DVD Industries, Londres, BFI.

Metz, Christian, 1972, Essais sur la signification au cinéma, tome 2, Paris, Klincksieck.

O’Toole, Peter, 2001, « Avertissement - édito », DVDvision, $\mathrm{n}^{\circ}$ 2, mai 2001, p. 3.

Rockstar Games, 2018, Red Dead Redemption II : le guide officiel complet, édition collector, Piggyback Interactive Limited.

Strauss, Frédéric, 2013, « Le marché du DVD est-il KO ? ", Télérama, 6 août 2013, [En ligne], https://www.telerama.fr/cinema/le-marche-du-dvd-est-il-ko,100876.php.

\section{NOTES}

1. «Quel sera le nouveau moyen d'enregistrement et de conservation des images ? [...] Un spectacle de plusieurs heures se conservera sous un faible volume. Disque, ou fil en bobine, ou sphère, ou feuilles reliées, il sera aisément maniable et permettra à l'amateur de conserver chez soi ses spectacles préférés [...]. Telle partie qui l'émerveille, il la fera passer cinq ou six fois de suite, découvrant à chaque coup une beauté nouvelle » (Barjavel 1994 : 64, cité dans Le Forestier 2009 : 159).

2. McDonald (2007), cité dans Bennett et Brown (2008b : 1).

3. Voir à ce propos notre article "Productivité et limites de la notion de "chapitre" au cinéma : classicisme des adaptations de la "qualité française" et pratiques réflexives contemporaines", issu du colloque "Construire le récit: histoire et poétique des chapitres » organisé en 2018 à Cerisy par Claire Colin et Aude Leblond, à paraître.

4. Il n'en demeure pas moins que ce travail portant sur les étapes de la production d'un DVD, qui demanderait à s'appuyer sur des témoignages d'acteurs du milieu, reste encore largement à faire, et excèderait notre ambition limitée ici à une problématisation introductive.

5. La réflexion amorcée ici mériterait en effet un examen approfondi de la notion de "découpage " au niveau théorique et historique, dans les différents sens qu'elle recouvre ou a pu recouvrir (voir les travaux de Laurent Le Forestier, notamment Le Forestier 2018). Notons que pour India Song (1974), premier film de Marguerite Duras édité par Benoît Jacob Vidéo (2005) dans une collection sous-titrée «Édition vidéographique critique ", la section du menu qui nous intéresse ici est nommée, dans le cas d'un tel film radical, «Chapitres et découpage » : chaque chapitre, dépourvu de titre, correspond invariablement à cinq plans numérotés.

6. Voir, entre autres exemples, le DVD du film Crash (David Cronenberg, 1996), édité en 2001 par StudioCanal, dans lequel le choix des extraits qui défilent en boucle sur les pages de menu mettent en exergue des séries de plans particulières au sein de chaque chapitre qui exacerbent notamment la dimension érotique du sujet. 
7. Dans le DVD zone 1 du film Alien (1979) édité par 20th Century Fox Home Entertainment en 1999, l'un des premiers DVD qui a proposé à notre connaissance un menu intégré dans un espace de la diégèse filmique - un exemple plus récent et plus abouti est celui de Silent Hill Revelation (Metropolitan Film \& Video, 2013, édition 2 disques) où, afin d'imiter la pratique vidéoludique, l'activation du "Menu interactif » conduit l'usager à devoir se déplacer dans l'environnement horrifique de la ville pour trouver la mention «Chapitrage »-, un seul des vingt titres de chapitre comprend des guillemets («Scene selection / Chapter 12 : “Here Kitty” »). Cependant, plusieurs autres titres du DVD d'Alien sont empruntés à une réplique de l'un des personnages (à l'instar de «Chapter 7: A Wonderful Defense Mechanism» ou «Chapter 13: No Blood, No Dallas»). On observe ici que l'intitulé du sommaire, reproduit dans le petit livret de cette édition spéciale ( 20 th Anniversary Edition»), est «Scene selection », terme utilisé par les éditeurs anglophones de manière sans doute encore plus fréquente que «Chapters » (ou « Chapter List »), même si les unités sont ensuite qualifiées, comme ici, de «chapitres » (il peut être aussi question de "scenes »). La notion de "sélection» rejoint celle de "chapitrage» en ce qu'elle affiche le fait que les "énoncés" (désignation des unités, éventuellement avec titres) sont le résultat d'une "énonciation» (une opération de délimitation des segments). Une réédition plus récente du même film Alien par le même éditeur, en 2011, témoigne de différences qui montrent combien le chapitrage proposé est le résultat de choix : le nombre total de chapitres a doublé (quarante) et huit d'entre eux citent entre guillemets une réplique (on retrouve la même phrase "No Blood, No Dallas" ", mais cette fois entre guillemets). La multiplication des chapitres tient sans aucun doute au statut de «film culte » d'Alien qui, avec les sequels, s'est encore renforcé dans l'intervalle auprès de fans de science-fiction qui en connaissent les moindres détails.

8. Précisons que les guillemets ne sont pas toujours de rigueur, comme dans le titre «T'as de beaux yeux » du chapitre 10 de l'édition par StudioCanal de Quai des brumes (1938). Sur le DVD de La Traversée de Paris (1956) édité par René Château en 2001, le titre des chapitres 2,4 et 5 qui reprennent une réplique proférée dans le segment concerné ne comporte pas de guillemets («Un peu de savon?»; " Janvier, 45, rue Poliveau », soit une citation d'une phrase répétée avec une intensité croissante par Grandgil interprété par Jean Gabin; «En route!", qui reprend une injonction proférée par Bourvil marquant une césure dans le récit filmique). Par contre, des guillemets figurent dans le titre du chapitre 6, "Salauds de pauvres », en raison du caractère transgressif de cette formule restée célèbre et que l'instance chapitrale ne tient sans doute pas à assumer.

9. Dans l'édition DVD 20th Century Fox de 2004, chaque film de la trilogie originale (1977-1983) de Star Wars est découpé en 50 chapitres, qui apparaissent sur un feuillet inséré dans chacune des trois boîtes. Par comparaison, notons que dans une page non signée intitulée "Conseils pratiques », les Cahiers du cinéma (2001: 8) font état d'une moyenne de quinze chapitres par film.

10. Par analogie avec le péritexte selon Genette (1987:11), nous considérerons que la partie du parafilmique située à proximité même du « texte » (en l'occurrence ici de son support physique, la galette sur laquelle le film est encodé) relève du péritexte filmique, alors que les suppléments du DVD par exemple (making of, entretiens) relèvent de l'épitexte. Notons que certaines composantes parafilmiques comme la publicité concernent simultanément le film et le DVD ; ainsi, la publicité pleine page pour le DVD de Mulholland Drive (David Lynch, 2001) qui occupe la deuxième de couverture du 
numéro hors-série des Cahiers du cinéma paru en novembre 2002 met-elle en exergue la présence d'un «chapitrage aléatoire basé sur les mystérieux objets du film...» (voir infra).

11. On peut noter sur ce point qu'Ugo Dionne qualifie les titres de chapitres «d'intertitres » (Dionne $2008: 366,381$ ).

12. À cet égard, l'énonciation chapitrale constitue une "couche» qui s'ajoute à une autre composante que nous avons proposé d'envisager en termes d'énonciation filmique (Boillat 2014) et qui relève également de l'étape de la diffusion du film: le doublage.

13. L'évolution de la prise en compte du support DVD est par exemple perceptible dans le magazine Video Watchdog spécifiquement consacré aux supports de diffusion de films appartenant au genre du fantastique, et ce dès la couverture : dans le premier numéro datant de 1990, les sujets annoncés en couverture sont les « raretés, changements de titres et restaurations "; une rubrique consacrée aux Laserdiscs apparaît en 1997 (numéro 37, annoncée en couverture dès le numéro 40); au numéro 42 , un bandeau annonce que les DVD seront aussi recensés, ce qui sera progressivement le cas ; en 2007 (numéro 130), le titre du magazine devient Video WatchHDog.

14. L'un des deux pôles identifiés par André Chaperon est le suivant: "un discours technophile qui non seulement fait passer au second plan la valeur intrinsèque des films [...] mais aussi s'essaie à refaire l'histoire du cinéma en faisant passer au premier plan les caractéristiques techniques originelles du film [...], dont les motivations esthétiques sont alors volontairement évacuées et qui sont mises au même niveau que la qualité du pressage » (Chaperon 2001a : 27).

15. Par exemple, l'auteur qui compare dans Video Watchdog la version "director's cut » de Alice, Sweet Alice (1976) avec la version originale du film note des coupes à la fin des chapitres 15 et 16 (Charles $1997: 47$ ).

16. URL : http://www.annees-laser.com, consulté le 9 décembre 2018.

17. «Pourquoi l'année 2004 a-t-elle marqué le summum du marché DVD ? Parce que c'est le moment où l'équipement en lecteurs a atteint son pic dans les foyers français » (Strauss 2013).

18. Le «V $\mathrm{V}$ " de la marque déposée « DVD » s'est d'abord et avant tout imposé pour qualifier la "vidéo» (dans le sens restreint de «film»), même si les fabricants ont labellisé le terme anglais « versatile » pour signifier au contraire la possibilité d'utiliser ce moyen de stockage pour tout type de données numériques.

19. Pour un constat posé en 2001 quant à la faible valeur ajoutée offerte par les bonus, voir Chaperon 2011a.

20. On lit par exemple dans Les Années laser à propos du DVD de Twilight I: Fascination qu'il comporte un "making-of en 7 chapitres thématiques classiques à base d'entretiens consensuels» ou à propos d'une version collector de Iron Man qu'on y trouve un «making-of panoramique, divisé en chapitres chronologiques». URL: http://www.annees-laser.com/content/view/526/29/; http://www.annees-laser.com/ content/view/343/27/ (consultés le 9 décembre 2018). Notons que le descriptif technique des DVD ne comprend pas la mention de l'éditeur, ce qui en dit long sur l'intérêt accordé à son travail sur le périfilmique.

21. Django Unchained (Quentin Tarantino, 2012), Sony Pictures Home Entertainment, boîte en métal «SteelBook», DVD+Blu-Ray, 2013. 
22. Dans son célèbre article «Le cinéma d'attraction : le film des premiers temps, son spectateur, et l'avant-garde", Tom Gunning mentionne en passant que "le cinémaspectacle a clairement réaffirmé, dans un passé récent, son ancrage dans la culture de la stimulation et les tours de montagnes russes - je pense plus particulièrement à ce que l'on pourrait appeler le cinéma d'effets du trio Spielberg-Lucas-Coppola » (Gunning 2006). On peut penser aussi aux attractions plus ou moins autonomisées du récit que proposent les comédies musicales ou les genres apparentés, dont les éditions DVD optent souvent pour des titres de chapitre correspondant à ceux des numéros chantés. Ainsi, au sommaire du DVD de Chantons sous la pluie (Warner, 2002, édition spéciale 2 disques), 12 des 38 titres de chapitres figurent en italique car ils reprennent le titre du morceau chanté.

23. Dans le cadre de la pratique éditoriale discutée ici, voir l'analyse par Tom Brown (2008) du DVD «édition spéciale » du film d'animation Le Roi Lion commercialisé par Disney, que le chercheur examine - en particulier sous l'angle des bonus qui nous font accéder aux coulisses de la création infographique du film et du «contrôle environnemental » qui rapprocherait le DVD du parc à thèmes - à l'aune des caractéristiques du « cinéma des attractions » relevées par Tom Gunning. Le chapitrage qui met en valeur des moments spectaculaires est discuté (84-86).

24. Avec le Blu-ray, on observe une tendance au remplacement du modèle du sommaire par une ligne de temps sur laquelle se déplace un curseur, selon un modèle emprunté au visionnement en streaming (notamment sur YouTube). Ainsi, par exemple, alors que les Blu-ray des six premiers volets de la série des Fast and Furious édités par Universal comportent la rubrique "Les chapitres » dans leur menu, les deux derniers opus édités en 2017 se contentent d'une icône représentant un livre ouvert. Notons que le Blu-ray est lu par certains usagers à partir d'une console de jeu vidéo, ce qui tend à atténuer la perception d'une distinction entre les médias. Il serait d'ailleurs intéressant d'explorer la notion de "chapitre " dans l'histoire des productions vidéoludiques. Le récent Red Dead Redemption II (Rockstar Games, 2018), qui se réfère constamment au western cinématographique, est par exemple structuré en six chapitres (suivis d'un épilogue en deux parties) qui viennent ponctuer le développement du récit (cadrage d'autant plus notable qu'il s'agit d'un jeu à monde ouvert). Le volumineux manuel vendu parallèlement au jeu consacre une centaine de pages aux diverses segmentations d'une partie de jeu, qu'il présente ainsi : «L'histoire de Red Dead Redemption II est divisée en chapitres. Chacun compte un certain nombre de missions, qui se débloquent selon un ordre et des conditions spécifiques. [...] Pour vous aider à y voir plus clair, chaque section de ce cheminement débute ainsi par un grand diagramme [...] qui recense toutes les missions du chapitre concerné [...]. Pour une clarté optimale, le texte est découpé en paragraphes numérotés; chaque chiffre est reproduit sur la carte du secteur » (Rockstar Games 2018 : 30). Cette présentation montre bien comment, dans un jeu où l'espace à arpenter peut primer sur la temporalité du récit, la linéarité de l'histoire chapitrée peut être maintenue tout en étant transposée sur un plan topographique.

25. On pense aux DVD bon marché ou à ceux où le travail éditorial spécifique au DVD, qui consiste parfois en la reprise minimale d'un catalogue antérieur de copies VHS (comme chez René Château), est quasi nul. Dans de tels cas, l'entièreté du film peut tenir sur une seule plage, ou les balises peuvent être introduites indépendamment du contenu du film, comme dans la collection à jaquette rouge «Gaumont à la demande » 
qui compte quelque 250 titres rares du patrimoine français, où la page de menu des DVD précise qu'en utilisant la touche munie d'une flèche de la télécommande, l'usager peut «navigue[r] dans le film par intervalle de 10 minutes». Notons que pour des éditions plus prestigieuses, telles que celle du Rouge et le Noir (1954) comportant deux disques respectivement consacrés à chacune des "parties » du film souhaitées par le cinéaste Autant-Lara, Gaumont propose un chapitrage.

26. Dans les mêmes livrets de la série des Antoine Doinel éditée en 2001-2002 (ces pages seront supprimées lors de rééditions ultérieures), Toubiana précise à propos des films de «Revoir François Truffaut »: «[L]e support [DVD] permet d'envisager chaque film accompagné de divers compléments : images d'archives, films commentés, découpage en chapitres, bandes-annonces. " Notons que pour les films de Truffaut, le sommaire "Chapitres du film» est imprimé dans le livret en regard des «Chapitres du commentaire » qui segmentent le discours réflexif émis sur l'une des pistes son par un collaborateur du film, par exemple avec le scénariste Jean Gruault pour Les Deux Anglaises et le continent (MK2, 2000). Ce dédoublement du chapitrage pour un même film est révélateur d'un souci de favoriser une consultation partielle dans une optique informative, l'opération de segmentation représentant en elle-même une forme de discours sur le film.

27. Plus tard, par exemple dans l'édition de 2011 de Fahrenheit 451, l'interactivité du chapitrage ne sera plus mentionnée par MK2 au verso de la jaquette; en ce qui concerne les films de Kiarostami, on peut signaler que pour le DVD de Like Someone in Love (2012) proposé par le même éditeur l'année même de la sortie du film, les chapitres sont munis d'un matricule mais dépourvus de titres.

28. Le Forestier fait mention d'un "rétrécissement temporel ", « dans la mesure où le DVD accentue les possibilités et le désir d'interruption du flux filmique (pour passer au chapitre précédant ou suivant, pour accéder à un autre aspect du contenu, etc.) » (Le Forestier $2010: 163)$.

29. Comme nous l'indiquions en ouverture d'un article consacré au film : «La chaîne Canal+, coproductrice de Mulholland Drive, a même proposé à l'occasion de la première diffusion du film sur ses ondes un court métrage explicatif (Retour à Mulholland Drive, réalisé en 2003 par Philippe Rouyer, critique à Positif) censé livrer les clés requises pour une "bonne" compréhension de l'œuvre " (Boillat $2005: 48$ ).

30. Pour le film en deux volets à récit arborescent Smoking/No Smoking (1993), l'éditeur (Pathé Vidéo, 2004) propose une avancée linéaire dans les chapitres, mais offre la possibilité à l'usager lorsqu'il se trouve à l'un des embranchements du récit d'opter pour une alternative donnée (entre deux et six bifurcations narratives identifiées par le titre indiqué dans le film sur un carton pourvu d'une illustration dessinée).

31. Certains d'entre eux sont d'ailleurs communs à plusieurs entrées de chapitre, ce qui met en péril la fonction organisationnelle du chapitrage. Dans l'édition Blu-ray du même film dans la collection "StudioCanal» (2010), ce principe a été conservé, et le nombre d'extraits par chapitre a même été accru.

32. Il faut remarquer en effet que le cinéma de Lynch a incarné par excellence l'objet du fétichisme cinéphilique à la française ; l'édition MK2 (2004) de Fire Walk With Me (1992) incluait même dans chaque boîte cartonnée un "morceau de pellicule originale numéroté ». 
33. Le chapitrage porte en fait à la fois sur le film et sur l'œuvre du cinéaste, puisque quelques rares extraits du «Lynch-kit » sont tirés de courts-métrages, et non de Inland Empire; il s'ouvre ainsi à l'intertextualité.

34. Ou d'un autre type de production médiatique. Par exemple, au vu de l'importance structurelle et culturelle du chapitrage dans le roman graphique Watchmen (voir Boillat 2018), sa suppression dans le film homonyme de Snyder puis sa réinsertion dans le menu du DVD est à noter (en l'occurrence, les 43 chapitres de l'édition Paramount ne sont aucunement pensés par rapport au découpage originel en 12 chapitres).

35. Les premières versions des intégrales de chaque saison de House of Cards diffusées par Sony ont été divisées en deux volumes par saison (pour des raisons sans doute strictement commerciales), tandis que chaque «épisode " muni d'un matricule y est qualifié de "chapitre» tant sur la jaquette de la boîte que dans le menu (bien que l'entrée principale s'intitule «Episode Selection»). On peut se demander si le terme même de "chapitre » joue un rôle dans l'appréhension du récit par les scénaristes; dans le premier chapitre de la deuxième saison (2014), Francis Underwood (Kevin Spacey) propose à la journaliste Zoé Barnes (Kate Mara) d'« entamer ce nouveau chapitre en partant de zéro » (23:43-23:47) ; cette réplique prend un relief particulier puisque, à la grande surprise des téléspectateurs, il assassinera Zoé Barnes plus tard dans le même épisode.

36. Même si les unités délimitées par des cartons ne sont pas explicitement qualifiées de chapitres, comme dans Cléo de 5 à 7 (Agnès Varda, 1962) ou Elephant (Gus van Sant, 2003).

37. Le DVD d'Elephant offre un chapitrage non linéaire qui permet d'entrer dans le film par chaque prénom de personnage, soit en accédant à un chapitre défini en amont par la mention écrite qui apparaît dans le film, soit en déplaçant un curseur sur une représentation cartographique $d u$ lieu du drame et en sélectionnant des étapes du parcours de chacun des personnages (ou couples de personnages). Cette conception topographique de la diégèse n'est pas sans incidence sur la lecture que l'on peut avoir du film. L'édition plus récente en Blu-ray coéditée par MK2 et TF1 Vidéo présente quant à elle un découpage classique en douze chapitres numérotés et dépourvus de titre.

38. Le cas du Redoutable est particulier en ce que le chapitrage y fonctionne comme une référence au cinéma de Jean-Luc Godard dans les années 1960, dont le film raconte l'histoire. À ce titre, les DVD des films de Godard seraient à aborder comme un corpus spécifique, tant leurs segmentations ostensibles et souvent erratiques - le découpage y est pratique de rupture - ont des incidences sur le travail des éditeurs de DVD.

\section{RÉSUMÉS}

Le présent article entend esquisser une réflexion pour une étude de l'usage du chapitrage dans le domaine de l'édition de films en DVD, un support certes aujourd'hui sur le déclin mais qui a connu une diffusion massive, et qui a largement contribué à introduire et à populariser un changement radical par rapport au défilement continu que proposait avant lui la VHS. Le 
chapitrage, qui oriente la lecture du film dans le cas de re-visionnages, peut être plus ou moins valorisé et présenté de différentes manières par l'éditeur, avec des effets qui sont discutés ici, à partir d'exemples variés, en tenant compte de la discontinuité foncière du médium cinématographique. Une approche comparative est également prônée qui envisage le découpage en chapitres du DVD en fonction de l'éventuelle segmentation explicite du film en partie, voire du roman dont le film est une adaptation. À l'image des couches du support, on peut observer comment ces différentes configurations chapitrales coexistent dans certains cas en une forme de palimpseste.

This article aims to outline a reflexion for a study of the use of chaptering in the field of DVD film publishing, a medium that is now in decline but that has introduced and popularized a radical change from the continuous viewing required before by VHS. Chaptering, which guides the reading of the film in the case of re-visioning, can be more or less valued and presented in different ways by the publishers, with effects that are discussed here taking into account the fundamental discontinuity of the film medium. A comparative approach is promoted by envisaging the splitting of a DVD into chapters according to the possible explicit segmentation of the film in parts, or even to the chapters of the novel of which the film is an adaptation. Like the layers of the support, we can observe how these different chapter configurations coexist in some cases in a form of palimpsest.

\section{INDEX}

Keywords : chapter, cinema, DVD (publishing), film editing, narration, interactivity, intermediality, cinephilia

Mots-clés : chapitre, cinéma, DVD (édition), montage, narration, interactivité, intermédialité, cinéphilie

\section{AUTEUR}

\section{ALAIN BOILLAT}

Université de Lausanne 